\title{
EN DEFENSA DE LOS NIÑOS. LA BATALLA POR LA LACTANCIA MATERNA EN LA INGLATERRA DEL SIGLO XVII
}

\author{
On Behalf of Children. The Struggle for Breastfeeding in Seventeenth- \\ Century England
}

\author{
Antonella CaGnolati \\ Università degli Studi di Foggia \\ a.cagnolati@unifg.it
}

Fecha de recepción: 16-II-2013

Fecha de aceptación: 27-II-2013

Resumen: En la Inglaterra del siglo XVII se generó un debate sobre la lactancia materna. Todas las fuentes se centraban en el carácter sagrado de la procreación y en el hecho de que los deberes de una madre no se limitaban a dar a luz al niño, sino que también se procuraba su bienestar, por lo que su crecimiento era saludable en lo físico y lo espiritual. En consecuencia, entre los imperativos que debía respetar una buena madre estaban la lactancia materna, la atención a la limpieza y la higiene del cuerpo del bebé, la preocupación vigilante para los tiempos difíciles del crecimiento, del destete y la enfermedad. Sin embargo, los hábitos diferían entre las familias ricas que habían optado por contratar a una niñera pues eran, sin distinción de ningún tipo o clase, comerciantes, abogados, médicos y aristócratas. La costumbre exigía que el bebé se alojara en la casa de la niñera, a menudo a varios kilómetros de distancia de la casa del niño. Para permitir que el bebé pasase los primeros meses de vida en un entorno considerado más saludable, con suficiente antelación a la fecha prevista para la entrega, el futuro padre contrataba a una mujer sana y bien alimentada. De hecho, a menudo las condiciones físicas de la niñera y el medio ambiente en el que vivía estaba lejos de ser idílico: el resultado fue una tasa de mortalidad infantil muy alta. Algunos empezaron a oponerse a este sistema con razones válidas.

Palabras clave: Lactancia, niñera, mortalidad infantil, deberes maternales, salud infantil, tratados de medicina, consejos para la nuevas madres. 
AвSTRACT: In the 17th century England a discussion on breastfeeding began. All sources emphasized the sacredness of procreation, affirming that the duties of a mother were not limited only to give birth to the child, but to concentrate on its welfare, and on its physical and spiritual healthy growth. Consequently, among the imperatives that a good mother should respect were primarily breastfeeding, attention to cleanliness and hygiene of the baby, watchful concern for weaning and diseases. However, the habits were really different: rich families, without distinction of any kind or class, merchants, lawyers, doctors, aristocrats, always hired a wet nurse. Traditional habits demanded that the baby stayed at the wet nurse's house, often several miles away from the child's home. To allow the baby to pass through the first months of life in a healthier environment, well in advance of the expected date of delivery, the father hired a healthy and well fed woman. In fact, the physical conditions of the wet nurse and the environment in which she lived were often far from idyllic: the result was a very high infant mortality rate. Some began to oppose this system with valid reasons.

Keywords: Breastfeeding, wet nurse, infant mortality, maternal duties, child health, medical treatises, advices for the new mothers.

\section{PREÁMBULO}

En el siglo XVII el debate sobre la lactancia materna interesaba a médicos, teólogos y comadronas y por eso se afrontaba desde dos puntos de vista distintos: bajo un perfil social, como hábito consuetudinario dotado de una larga tradición, y a nivel religioso $^{1}$. Casi todos los manuales de obstetricia, que proporcionaban embrionarios consejos de puericultura, recomendaban que las madres criaran a su hijo ${ }^{2}$ con la propia leche, pero en los tratados también se reflejaba una postura ambigua: aunque defendían la «naturalidad» de la leche materna, al mismo tiempo los médicos daban consejos concretos e instrucciones detalladas para elegir a una buena nodriza, y señalaban especialmente la necesidad de comprobar sus dotes morales, que podían tener graves consecuencias en el crecimiento psicológico del niño, en lugar de indagar sobre sus cualidades físicas de constitución sana y robusta.

A un nivel bastante realista los médicos consideraban válidos y compartían en gran medida los motivos sociales y económicos que impulsaban a las mujeres de la época a confiar sus pequeños a las nodrizas, aunque a nivel teórico todos estaban de acuerdo

1 Cfr. VALERIE Fildes, Wet Nursing. A History from Antiquity to the Present, Oxford, Blackwell, 1988. (En concreto el Capítulo 6: "The English Wet Nursing System in the Seventeenth Century», pp. 79-100).

2 Cfr. Alice Judson Ryerson, «Medical Advice on Child Rearing, 1550-1900», Harvard Educational Review, 3, 31, 1961, pp. 302-323; Patricia Crawford, "The Suckling Child': Adult Attitudes to Child Care in the First Year of Life in Seventeenth-Century England», Continuity and Change, 1, 1986, pp. 23-51; Joseph E. Illick, "Child-Rearing in Seventeenth-Century England and America», in Lloyd DeMause (Ed.), The History of Childhood, New York, The Psychohistory Press, 1974, pp. 303-350. 
en la superioridad de la lactancia materna. Los prejuicios que obstaculizaban la difusión de esa práctica estaban bastante arraigados y reprimían a las clases nobles y a la gentry (alta burguesía): pues parecía que era muy poco conveniente para una dama someterse a los esfuerzos físicos de la lactancia, especialmente porque tendría que afrontar las severas críticas de los familiares, las rémoras de su consorte y la desdeñosa exclusión de su clase social. Para conocer los motivos que podían convencer a las mujeres a dar de mamar a sus propios hijos es interesante la «Preface to Ladies, wherein they are exhorted to nurse their Children themselves» en The Nursing of Children de J. Guillemeau ${ }^{3}$. El autor deja claro su punto de vista desde las primeras líneas, afirmando que

[No existe] diferencia alguna entre una madre que se niega a criar a su propio hijo y otra que lo mata una vez que ha sido concebido, con el fin de no soportar el engorro de llevarlo nueve meses en su vientre. Así pues, ¿por qué una mujer con esa justificación se niega a criar a su hijo con su sangre, en el interior de su vientre, así como se niega a darle su leche una vez que ha nacido ${ }^{4}$ ?

Adicionalmente, en el prefacio se debaten y argumentan con detalle otros cuatro motivos a favor de la lactancia materna: en primer lugar, Guillemeau plantea a las madres el peligro de que el hijo que han engendrado con grandes esfuerzos y riesgos pueda ser intercambiado por la nodriza, ante la eventualidad de que el bebé estuviera muerto o fuera mutilado por los lobos o por otros animales. Después está la defensa de los lazos afectivos que se establecen entre madre e hijo en el momento de la lactancia, un afecto que se consolida durante la vida y que se perdería o pasaría a la nodriza en lugar de a su madre natural. Aún más presente estaba, entre los prejuicios de la época, el temor de que la «disposición natural» de la nodriza, es decir, sus inclinaciones y su índole llegaran al niño a través de la leche y contribuyeran en gran manera a conformar el carácter del recién nacido. Por último, la nodriza podía transmitir al niño algunas enfermedades que le harían más débil y que le marcarían, una vez que fuese adulto, para el resto de su existencia.

Se puede argumentar que Guillemeau no presenta ninguna prescripción realmente «médica», sino que se limita a señalar nociones que estaban muy presentes en

3 Jacques Guillemeau, Child-Birth, or the Happy Delivery of Women. Wherein is Set Downe the Governement of Women, London, J. Norton, London, 1635. La segunda parte del tratado titulada The Nursing of Children tiene que ver con la crianza de los niños, desde su nacimiento hasta el destete. Utilizo el texto en inglés porque su difusión en el ambiente de los médicos y comadronas fue amplia y porque no existe, hasta finales del siglo XVII en Inglaterra, un conjunto de tratados autóctono. El volumen de Guillemeau vuelve a proponer topoi ampliamente difusos en la cultura de los médicos de la época, válidos también para Inglaterra. Véase también VALERIE Fildes, «Infant Care in Tudor and Stuart England", Midwife, Health Visitor, Community Nurse, XXII, mar. 1986, pp. 79-80; 82-84.

4 Jacques Guillemeau, cit., sig. Ii $^{\mathrm{v}}$. 
el sentimiento común de la época, sacadas del ámbito ético (la relación afectiva entre madre e hijo, la posibilidad de un crecimiento moral torcido) y social (el temor por un "cambio» del propio recién nacido o por la muerte del bebé). Sin embargo, a pesar de que esta premisa indica un sentimiento común de los médicos sobre la lactancia materna y de que conocen su superioridad frente a las tareas de las nodrizas, todo el tratado gira paradójicamente en torno a la voluntad del autor de dar consejos inteligentes a las madres para elegir a una buena nodriza.

Para menoscabar el muro del prejuicio social era necesario apelar fehacientemente a los motivos religiosos: ésta fue la innovación argumentativa de los Puritanos que definieron rigurosamente la práctica de la lactancia materna como la función prioritaria de una buena madre, exaltando el sentido del deber intrínseco a esa acción y añadiendo que dicho comportamiento habría sido recompensado y bendecido por Dios 5 .

\section{LA BREASTFEEDING COMO DEBER DE UNA BUENA MADRE}

En los tratados que tienen por objeto el household government $t^{6}$, encontramos sin excepción una parte importante relativa a los «deberes» de la buena mujer, codificados dentro de su relación con el marido, con los hijos y con los empleados domésticos. Entre las obligaciones fundamentales siempre se incluye la práctica de la lactancia, que se justifica a través de las páginas de las Sagradas Escrituras.

En A Godly Form of Household Government ${ }^{7}$, Robert Cleaver define, con claridad lógica y argumentativa, las justificaciones que los Puritanos presentaban contra la crianza mercenaria: por tanto se van dilucidando gradualmente una serie de principios que serán retomados, acogidos y ampliados progresivamente en su fuerza compulsiva a través de las páginas de los tratados sobre la familia y en los comentarios al texto bíblico ${ }^{8}$.

5 Cfr. Robert V. Schnucker, «The English Puritans and Pregnancy, Delivery and Breast Feeling», History of Childhood Quarterly, 1, 1974, pp. 637-658.

6 Se trata de un tipo de texto que florece a finales del siglo XVI y que alcanza su máxima popularidad en los dos primeros decenios de 1600. En estas obras se recomendaban, con una precisión minuciosa, los comportamientos que cada uno de los miembros de la household debía adoptar.

7 Robert Cleaver, A Godly Form of Household Government: for the Ordering of Private Families, According to the Direction of Gods Word, London, T. Man, 1598.

8 Sobre el universo cultural de los Puritanos, véanse Patrick Collinson, The Elizabethan Puritan Movement, London, Methuen, 1967; Christopher Hill, Society and Puritanism in Pre-Revolutionary England, London, Secker and Warburg, 1964; James Turner Johnson, A Society Ordained by God. English Puritan Marriage Doctrine in the first half of Seventeenth Century, Nashville, Abingdon Press, [1970]; John Morgan, Godly Learning. Puritan Attitudes towards Reason, Learning and Education, 1560-1640, Cambridge, Cambridge University Press, 1988; Levin Ludwig Schucking, The Puritan Family: A Social History from Literary Sources, London, Routledge and Kegan Paul, 1969. Para la conformación de la identidad de la mujer puritana véase Antonella Cagnolati, Lo specchio delle virtù. Modelli femminili nell'Inghilterra puritana (1560-1640), Roma, Aracne, 2004. 
La función prioritaria de una madre de cara a sus hijos -afirma Cleaver- tiene que ver con el deber de criarles: el incumplimiento de esa obligación se configura como un pecado contra la «ley natural» y contra la «voluntad de Dios»". Adicionalmente, se señala que las funciones de la nodriza son peligrosas para la salud física y mental del pequeño, y también para su propia madre ${ }^{10}$.

La «ley natural» manifiesta claramente que los animales también son criados por quien los ha engendrado, en cambio a veces las mujeres «adoran ser madres, pero no criar a sus hijos». En segundo lugar, para reforzar más que esa práctica resulta conforme a la voluntad divina, se citan páginas de la Biblia que muestran casos evidentes de mujeres que han obedecido a Dios rigurosamente, dando de mamar a sus propios hijos: Eva que educó a Caín, a Abel y a Set; Sara que crió a Isaac; Ana que dio su leche a Samuel y, como ejemplo poco discutible, el de la Virgen María ${ }^{11}$.

Cleaver plantea también una conexión entre la función de criar a los hijos con la leche materna y la fama que se cierne en torno a una mujer cuando ella ya se encuentra mayor: «es un elogio para una buena mujer [...] si ha criado a sus propios hijos».

Así se definen algunos criterios que relacionan correctamente la lactancia-acción como algunas otras de tipo solamente fisiológico-con las virtudes morales de una mujer: si ella presenta un rechazo hacia esta importante tarea, se reconoce culpable de haber infringido "el sagrado vínculo de la naturaleza» y la ley de Dios, porque este Dios ha dado a la mujer el seno para que críe al niño.

Esas mismas justificaciones se encuentran en Christian Oeconomie ${ }^{12}$ de William Perkins, ya no sólo colocadas dentro de largas digresiones sobre las tareas de la buena madre sino entre los deberes de los padres, indicio de que la salud y el bienestar del recién nacido tendrían que haber importado mucho a ambos cónyuges, planteando así un rol activo del padre en los cuidados que debía dedicar al pequeño.

En las páginas de Perkins se entrelazan reclamos bíblicos y referencias culturales, pero lo que parece nuevo es el reclamo intencional al marido para que dé fuerzas y apoye

$\overline{9}$ «Among the particular duties that a Christian wife ought to performe in her familie, this is one: namely, that shee nurse her owne children: which to omit, and to put them forth to nursing, is both against the law of nature, and also against the will of God», Robert Cleaver, A Godly Form of Household Government: for the Ordering of Private Families, According to the Direction of Gods Word, cit., p. 235. (La cursiva es propia).

10 Siguen en el texto los tradicionales motivos que, con tonos bastante negativos, consideran la índole y el carácter de la nodriza y sus posibles repercusiones sobre el niño como nefastos.

11 Eva: Génesis, 4; Sara: Génesis, 21, 6-7; Ana: 1 Samuel, 1; María: Evangelio según San Lucas, 2, 6-7.

12 William Perkins, Christian Oeconomie: or, A Short Survey of the Right Manner of Erecting and Ordering a Familie, According to the Scriptures, London, F. Kyngston, 1609. Para las alusiones al deber de lactancia materna, véanse las pp. 134-145 (Capitulo XIII: «Of Parents»). 
a la consorte en la tarea de dar de mamar a su hijo: así pues Perkins parece haber identificado plenamente el gran obstáculo que impedía a la mujer cumplir con una práctica tan natural, es decir, las necesidades sexuales y relacionales con el cónyuge, quien prefería confiar el pequeño a una nodriza y poder disponer de nuevo de su mujer con fines reproductivos. Perkins da un vuelco a este prejuicio al implicar a los maridos en esa acción y al hacerles plenamente responsables ante los ojos de Dios.

Un debate más analítico y convincente, fruto de una profunda reflexión teorética y de una notable experiencia personal, se encuentra en Of Domesticall Duties ${ }^{13}$ de William Gouge. Dentro de los ocho arduos tratados que componen el libro de más de seiscientas páginas, Gouge dedica un apartado importante a la lactancia materna, haciendo una identificación exhaustiva que toma muy en cuenta tanto las posturas ya adoptadas en gran medida por la predicación puritana, como las tradiciones culturales asumidas por los autores del clasicismo «pagano».

Gouge afronta la cuestión de la "lactancia» de manera omnicomprensiva, partiendo de una especificación minuciosa de las cautelas y atenciones que todas las madres deben tener durante el difícil periodo del embarazo. Los consejos que él ofrece se dirigen a proteger a la nueva criatura que nacerá y a implicar activamente, como ya había sostenido Perkins, a los maridos en el cuidado de las mujeres ante una circunstancia tan importante de la vida familiar, que ambos han construido juntos. Gouge no duda en condenar la práctica (evidentemente bastante usual entonces) del aborto, que se conseguía a través de substancias farmacológicas y el abandono de los pequeńos recién nacidos, considerando esos actos un verdadero crimen contra la ley ${ }^{14}$.

El debate sobre la lactancia y los motivos que deben impulsar a las mujeres a esta práctica parece exhaustivo y convincente. Según la lógica típica del conjunto de tratados puritanos, él identifica argumentos válidos que tienden a demostrar que la lactancia materna es un deber: se vuelve a proponer de nuevo la combinación de la "palabra de Dios» y las «leyes naturales» que concurren juntas para definir lo mejor posible «si las mujeres deben estar vinculadas a ese deber o no».

A través de una dialéctica articulada que se hace escudo en las páginas bíblicas y en los embrionarios conocimientos médicos, Gouge supera los argumentos elaborados anteriormente para establecer un principio sólido: la concesión de la leche materna es un

$\overline{13}$ William Gouge, Of Domesticall Duties, Eight Treatises, London, W. Bladen, 1622.

14 Véanse las siguientes afirmaciones al respecto: «They through violence of passion, whether of griefe, or anger, or through violent motion of the bodie, as by dancing, striving, running, galloping on horsebacke, or the like: or through distemper of the bodie, or eating things hurtfull, by eating too much, by too much abstinence, by too much bashfulnesse in conceiling their desires and longings (as we speake) cause any abortion or miscarriage, fall into the offence contrary to the forenamed dutie», William Gouge, Of Domesticall Duties, Eight Treatises, cit., p. 506. 
don de Dios y al mismo tiempo una bendición; por tanto todas aquellas que no se atienen a tal norma son lógicamente maldecidas y no tienen la gracia de Dios, ya que desobedecen su voluntad. Para apoyar aún más tal idea, Gouge añade que hay que concienciarse de que la esterilidad en la Biblia se presenta efectivamente como una maldición divina: por tanto, es absolutamente reprobable ir contra los dictámenes manifestados por la voluntad de Dios y no llevar a cabo una práctica tan natural como la lactancia materna.

La lógica argumentativa de Gouge es bastante interesante, hasta el punto de que algunas reflexiones que él hace van a encontrar su lugar en el conjunto de manuales médicos de la segunda mitad del siglo XVII: al unir directamente el hecho de dar de mamar a los propios niños con un deber ético-religioso, él ancla firmemente dicha acción en la prioritaria vocación materna y en el reconocimiento de la santidad de dicho comportamiento.

\section{LAS PALABRAS DE UNA MUJER}

Más allá de manuales de fisiología y tratados teológicos, en el panorama del debate abierto sobre la lactancia materna hay que señalar que la primera toma de posición orgánica sobre el caso es obra de una mujer, Elizabeth Knyvet Clinton ${ }^{15}$ que en 1622 (y por tanto al mismo tiempo que la publicación de Of Domesticall Duties) ofrece a las imprentas un breve pamphlet que lleva por título The Countesse of Lincolnes Nurserie.

Las opiniones que surgen sobre el texto ponen de manifiesto las claras e innegables tendencias puritanas de la autora, además de su participación atenta y reflexiva en la elaboración teórica que entonces se llevaba a cabo y en el análisis de las posturas que se iban confrontando. The Countesse of Lincolnes Nurserie con seguridad no presenta reivindicaciones de carácter médico, es más, el terreno en que se mueve parece eminentemente religioso y social; el tratado, además de constituir un testimonio relevante, despierta interés, ya que aquí aparece plenamente realizado aquel paso tan importante para la ética puritana entre la normatividad enfatizada por los predicadores y la intimidad de la conciencia femenina. Adicionalmente, con habilidad retórica y energía, Clinton llega a relacionar la lactancia materna no sólo con la voluntad de hacer cosa grata a Dios, sino con el desarrollo progresivo del afecto natural que todas las madres experimentan por sus hijos.

La lactancia materna pasa a formar parte de aquellas funciones concretas que intentan connotar la imagen de la buena madre, a su vez parte indispensable de la identidad femenina desde el punto de vista del Puritanismo. No sólo Clinton defiende

15 Para una biografía de Elizabeth Knyvet Clinton véase Ernest Caulfield, «Progress in Pediatrics. The Countesse of Lincolnes Nurserie», American Journal of Diseases of Children, XLIII, 1932, pp. 151162. 
con argumentos válidos dicha práctica sino que verifica la importancia fundamental del ejemplo: pues el tratado está dedicado a Briget, mujer de Teophilus (hijo de Elizabeth), cuyo comportamiento es bastante elogiado por ser un raro ejemplo de madre que se había atrevido a desafiar las convenciones sociales para poder cumplir con el deber de ofrecer «la dulce leche de [su] propio pecho a [su] niño» ${ }^{16}$. El valor del ejemplo se pone bastante en evidencia: si una dama de noble cuna, como Briget, había decidido dar de mamar, desafiando prejuicios ancestrales, seguramente otras mujeres se habrían sentido valientes para seguirla e imitarla, cambiando por tanto la tan criticada práctica de confiar los recién nacidos a nodrizas mercenarias. La "Dedication» ${ }^{17}$ que introduce el tratado está llena de elogios y palabras afectuosas para toda mujer joven que con gran valentía se atreve a enfrentarse a una costumbre socialmente aceptada y compartida, ya que al mismo tiempo dicha elección manifiesta plenamente su carácter devoto, humilde y su índole de buena cristiana.

Igual que para otras obras escritas por mujeres, también en el caso de The Countesse of Lincolnes Nurserie se precisa una aprobación masculina que haga la función de garantía para ser publicada. Aquí encontramos un "Prefacio» dirigido al lector donde Thomas Lodge ${ }^{18}$, personaje bastante polifacético, invita a la lectura del breve tratado y aporta algunos motivos interesantes. El intento de justificación es bastante manifiesto: Lodge explica que el motivo fundamental que ha impulsado a la autora a "poner el bolígrafo sobre el papel» reviste un alcance social, es decir que muestra su intención de criticar una «práctica innatural» que conduce a las mujeres a la degeneración moral. Lodge señala también la rareza del argumento, poniendo de manifiesto lo difícil que es para el «lector cristiano» encontrar en otro lugar un "tratado específico» sobre dicho argumento. Por último la pertenencia de la autora a la nobleza parece una garantía válida de todo el contenido de la obra.

Un impulso de tipo moral procedente del reconocimiento de un mal social seguramente impulsó a Elizabeth Clinton a escribir: en las palabras de Lodge, por lo demás, no hay ninguna referencia a una presunta «misión» de tipo religioso, sino que más bien se exalta la usefulness del texto, que podría instar a otras mujeres a sumarse a una práctica conductual que, según los cánones de la época, se juzga como bastante impropia.

La apasionada defensa del breastfeeding por parte de Elizabeth Clinton tiene orígenes lejanos que hay que buscar en su experiencia personal: ella efectivamente desde las

$\overline{16}$ «In giving the sweete milke of your owne breasts, to your owne childe». The Countesse of Lincolnes Nurserie, sig. A2 .

17 "To the Right Honourable, and approved vertuous Lady Briget Clinton Countesse of Lincolne», The Countesse of Lincolnes Nurserie, sigg. A $2^{\mathrm{r}}-\mathrm{A} 3^{\mathrm{r}}$.

18 Cfr. sub voce 'Thomas Lodge', Dictionary of National Biography, London, Oxford University Press, 1973, vol. XIII, pp. 61-66. 
primeras líneas apela a su experiencia como madre, que en el transcurso de su larga existencia había dado a luz a dieciocho niños (de los cuales habían sobrevivido bien pocos) y a las observaciones que había hecho sobre la relación entre madre y recién nacido. Sus afirmaciones son tajantes y no dan lugar a dudas:

desde el momento en que Dios ha querido complacerme dándome su bendición con el nacimiento de muchos nińos, y de esta forma me ha impulsado a observar las muchas vicisitudes que suceden a las madres y a sus pequeńos, he considerado justo abrir mi mente para debatir una importante cuestión que todas las mujeres cuya intención sea criar a sus hijos deberían considerar seriamente, y para expresar mi pensamiento lo más claro posible, hasta escribiendo sobre ese argumento todo lo que Dios quiera complacerse en dirigir mi mente. En pocas palabras, la cuestión que pretendo plantear tiene que ver con el deber de dar de mamar que deben cumplir todas las madres con sus hijos ${ }^{19}$.

El conocimiento de un imperativo superior está estrechamente ligado a un fuerte sentimiento de culpa que se pone de manifiesto en las páginas, cuando Clinton sostiene «no haber cumplido este [mi] sagrado deber» por una serie de motivos que se declaran expresamente y nos permiten sacar un retrato sobre los prejuicios que entonces eran profundamente compartidos en relación a la lactancia. Así pues, con extrema sinceridad ella sostiene que está plenamente convencida de que habría tenido que poner en práctica dicho comportamiento, pero los impedimentos que se lo habían obstaculizado se imputaban en primera instancia a la autoridad del cónyuge que había objetado toda una serie de rémoras y obligaciones sociales; en segundo lugar, a los malévolos consejos que la habían engañado, y por último que todavía no había emprendido, con la debida convicción, el camino de profundización ética y religiosa que había afligido su consciencia y, por tanto, ella nunca se había dado cuenta en su juventud de que la lactancia constituía un deber concreto en el ámbito del rol materno ${ }^{20}$.

¿Cómo poner remedio a una responsabilidad tan importante que, si no se cumple a favor de los propios hijos, hace a todas las madres culpables ante los ojos de Dios? Elizabeth intenta eximir su conciencia planteando tres acciones válidas: el necesario arrepentimiento por la ofensa provocada a Dios y la petición de su perdón; la mayor efusión de amor y cuidados hacia los propios hijos, para hacerles olvidar la falta de lactancia materna; y por último, el punto más útil de nuestro análisis, la voluntad de realizar una tarea de convencimiento a otras mujeres para prevenir un pecado de ese tipo. Al recordar

19 The Countesse of Lincolnes Nurserie, p. 1 (La cursiva es de E. Clinton).

20 «I knowne and acknowledge that I should have done it, and having not done it, it was not for want of will in my selfe, but partly I was overruled by anothers authority, and partly deceived by some ill counsell, and partly I had not well considered of my duty in this motherly office, as since I did, when it was too late for me to put it in execution», The Countesse of Lincolnes Nurserie, pp. 15-16 (La cursiva es de E. Clinton). 
que las mujeres mayores tienen el deber de instruir a las jóvenes ${ }^{21}$, ella exhorta encarecidamente a todas las mujeres a cumplir un acto de amor hacia los recién nacidos y por tanto a darles la leche materna desde su nacimiento, no permitiendo que una extraña (que no los ha llevado en su vientre materno), haga por dinero lo que una buena madre debe hacer por amor ${ }^{22}$.

El deseo de expiación se convierte en una crítica áspera contra la nobleza, la clase social a la que la misma Elizabeth pertenece. Las damas se niegan con desdeño a hacer suya esta importante función innata de la maternidad, se les aleja por razones frívolas, como el temor a que la lactancia pueda hacerles envejecer precozmente y ver de esta manera marchitarse su belleza, o bien el aburrimiento y fastidio provocados por la ropa, que debe ser cómoda para que sea apta para dar el pecho al pequeño. En fin, ellas a veces sostenían no tener leche suficiente o bien que sus pechos estaban completamente vacíos, y esta era una justificación que atraía las críticas de los predicadores puritanos:

Pero, ¿cuáles son las mujeres que tienen el pecho siempre vacío? Perdonad si pienso que es un poco como la gota: los mendigos no la sufren nunca; sólo las mujeres de ciudad o las damas están afectadas. En el capítulo 9 de Osea los pechos vacíos son un castigo divino [...]; pero ¿qué desafortunadas acciones pueden haber cometido ciertas mujeres para imputar a una maldición del Cielo la propia incapacidad de dar leche? Si, entonces sus pechos están vacíos como dicen, tendrían que ayunar y rogar para que se les quitara tal condena ${ }^{23}$.

Parece que dichas críticas no dańaban los modelos conductuales difundidos entre la nobleza inglesa. Las objeciones adoptadas por las jóvenes esposas de las clases más altas se hacen oír en muchos tratados del siglo XVII y por tanto debemos considerar que se trataba de topoi ampliamente compartidos, dotados de una fuerza compulsiva que venía de la antigüedad. Véase al respecto el acento apenado con el que Henry Newcome defendía la novedad revolucionaria de la que eran testigos algunas madres que -a pesar de formar parte de una categoría tan rica y privilegiada capaz de buscarse las mejores nodrizas del mercado- con gran fuerza y desprecio hacia la crítica en la que habrían podido incurrir, decidían dar de mamar a sus pequeños:

Las damas que, sustrayéndose a la costumbre que hoy prevalece, asumen el compromiso de dar de mamar por sí mismas a la prole, a veces deben afrontar comentarios desagradables y palabras sarcásticas por parte de numerosísimas damas que dificultan

21 Carta a Tito, 2, 3-5.

22 «Now, therefore love them so far as to do this office to them when they are borne, morew gladly for love sake, then a stranger, who bore them not, shall do for lucre sake». The Countesse of Lincolnes Nurserie, pp. 16-17 (La cursiva es de E. Clinton).

23 Henry Smith, op. cit., p. 41. 
dicha práctica [...]. Una dama que acepta ser nodriza, incluso de su propio hijo, hoy está tan pasada de moda y se la considera tan poco aristocrática que se puede comparar con el caballero que no bebe, no impreca y no maldice [...]. Y si, por casualidad, hubierais tenido la suerte de observar cuánta timidez y qué pudor se manifiesta en estos caballeros cuando les acontece ser objeto de insultos y de burla por parte de hombres depravados y viciosos de su misma clase [...], entonces podríais imaginar perfectamente la valentía y la resolución que necesitan las madres de la buena sociedad que, por el sólo hecho de dar de mamar a los hijos, se exponen al vilipendio y a la burla de las damas que, siendo la mayoría, carecen de voluntad para asumir dicha incumbencia ${ }^{24}$.

Si volvemos al análisis del tratado de Elizabeth Clinton, podemos comprobar lo fuerte que es la voluntad que impulsa a la autora a demostrar con copiosos argumentos la necesidad de que todas las mujeres están obligadas a satisfacer la obligación de criar a $s u$ hijo con $s u$ leche. En primer lugar el tratado se aparta de la tradición porque no trata de dar consejos específicos sobre la educación de los hijos ante la eventualidad de la muerte de su madre, sino que el público al que Clinton se dirige, de manera a veces dura y tajante, son solamente mujeres: por tanto se traza un modelo comunicativo que favorece el vínculo relacional dentro de una categoría de género y adquiere autoridad en virtud de la inteligencia superior de la autora que deriva de su edad y experiencia.

Resulta necesario señalar el importante y profundo reconocimiento que Clinton hace a la práctica habitual de la crianza, reconocimiento que se coloca en un contexto bastante más amplio relativo a la crítica social de un comportamiento que ella considera absolutamente despreciable ${ }^{25}$. Las polémicas críticas se dirigen hacia dos ámbitos diferentes: in primis, se enfatizan plenamente las desviaciones ético-conductuales que conducen a las mujeres a lo que Clinton define como una grave desobediencia frente a la voluntad divina; en segunda instancia la crítica se convierte en un fuerte j'accuse al conformismo social de la época -hecho que me parece un deber darle la justa visibilidad-y se lanza para convertirse en una embrionaria reflexión de tipo económico.

Clinton no se ahorra expresiones especialmente negativas dirigidas a las mujeres de la nobleza: a la obediencia que ellas deben a Dios y a sus inderogables preceptos, ellas prefieren «sus placeres personales», aventurándose así sobre el peligroso sendero que conduce primero al rechazo y después al desprecio de la voluntad divina.

¿Cómo pueden esas madres, se pregunta la autora, no comprender que su comportamiento es incorrecto ante los ojos de Dios? Quizás sus escasas capacidades intelectuales

24 Henry Newcome, The Compleat Mother or, An Earnest Persuasive to all Mothers (especially those of Rank and Quality) to Nurse their own Children, London, J. Wyat, 1695, pp. 8-9.

25 The Countesse of Lincolnes Nurserie parece, bajo este perfil, acercarse mayormente a los tratados puritanos sobre la household government que a los tradicionales mothers' advice books. 
no les permiten comprender lo que están haciendo porque, en caso contrario, su conciencia se constituiría en un aviso tan fuerte que no les daría tregua ${ }^{26}$ alguna.

En el transcurso de la argumentación se traza una secuencia de negatividad que Clinton denuncia sin disimulo alguno, como por ejemplo la presunción exclusivamente femenina de anteponer sus deseos pecaminosos a las directivas divinas, o la ignorancia de aquellas mujeres que no habían contemplado, entre los deberes de cualquier mujer buena, la obligación recogida en la primera y más importante ordenanza divina contenida en el «creced y multiplicaos» (Génesis, 1, 22), en la que se suman perfectamente los intentos divinos de creación con el orden de la naturaleza ${ }^{27}$.

Para hacer más convincente aún la apelación en defensa del breastfeeding, Clinton recurre al valor emblemático de los ejemplos sacados de las Sagradas Escrituras, recuperando las vivencias relativas a cuatro figuras femeninas importantes: Eva, madre de todos los seres vivientes; Sara, mujer de Abraham; Ana, madre de Samuel y, por último, la Virgen María. La lactancia materna, bien documentada en las páginas de la Biblia, fue considerada por estas mujeres como un deber sagrado a cumplir con alegría, siendo plenamente conscientes de hacer una cosa grata al Señor y garantizar al mismo tiempo la salud a su hijo. Clinton hace un uso instrumental y propagandístico de esos ejemplos con bastante argucia: al considerarlos ampliamente como paradigmas de un comportamiento difuso en "tiempos antiguos», la autora contrapone el pasado, en el que la lactancia materna era una costumbre habitual, a los tiempos presentes, corruptos y depravados, y emite una inapelable sentencia de carácter moral que pone de manifiesto la negatividad del presente a favor de una presunta superioridad del pasado, bajo el perfil ético-religioso.

Como se comprende fácilmente, el paso siguiente en la estructura argumentativa del tratado se centra en una crítica con más acento social: Clinton desaprueba, con tonos ásperos, la conducta de la nobleza porque considera, justamente con razón, que sus modelos conductuales son imitados por las clases subalternas. En lugar de dar ejemplos de virtud, las damas nobles fomentan comportamientos torcidos hasta en aquellas que quizás siempre habían dado el pecho por causas de carácter económico, pues no tenían suficientes recursos para pagar a una nodriza.

Otros dos motivos sostienen la tesis de Clinton: en primer lugar, ella señala la no fiabilidad de las nodrizas, cuyas características había tenido la oportunidad de observar bien: aunque algunas se mostraban atentas a la salud del pequeño y parecían diligentes

26 "Oh what peace can there be to these womens consciences, unlesse through the darknes of their understanding they judge it no disobedience?». The Countesse of Lincolnes Nurserie, p. 2.

27 "Of these I demand why then they did venture to marry and so to bear children?». The Countesse of Lincolnes Nurserie, p. 14. 
y eficientes, la mayoría sin embargo eran vagas, a veces maltrechas de salud y -un hecho aún más dramático-, se desinteresaban de los niños que se les confiaban hasta el punto de que, afirma la autora, "temo que la muerte de uno o dos de mis hijos derive de la negligencia de sus nodrizas $»^{28}$.

Por último, en la crianza está implícita una amenaza contra el orden social que deriva de motivos económicos: las mujeres pertenecientes a clases más pobres están obligadas a alejar de sí a sus niños para ocuparse de los hijos de una dama más rica, negando al mismo tiempo el afecto materno al pequeño que han llevado en su vientre.

Así pues, parece que entre las líneas de The Countesse of Lincolnes Nurserie la lactancia mercenaria se perfila claramente como un comportamiento a condenar, no sólo por motivos religiosos que se nutren de la apelación a modelos bíblicos ejemplares, sino en virtud de las nefastas repercusiones de carácter social que provocan consecuencias absolutamente criticables.

Un análisis en su conjunto reconduce nuestra reflexión a los deberes que -por derecho- son considerados fundamentales y constitutivos de una buena madre, icono difuso de sabiduría, buen sentido, afecto y devoción. Para evidenciar lo fuerte y enraizada que estaba ya en 1600 dicha imagen, voy concluir el estudio con las emotivas palabras pronunciadas por Nicholas Guy con motivo de las exequias de Elizabeth Caulton, mujer del predicador puritano William Gouge, desaparecida a la edad de cuarenta ańos, pocos días después de haber dado a luz su decimotercer hijo:

Así quiso Dios llevársela consigo, justo cuando ella estaba en lo mejor, cumpliendo su vocación de madre, en el momento del parto, cuando se puede decir que la muerte de una mujer equivale a la muerte del soldado que cae en una batalla, o bien al predicador que concluye su existencia en el púlpito ${ }^{29}$.

$\overline{28}$ The Countesse of Lincolnes Nurserie, p. 18.

29 Nicholas Guy, Pieties Pillar: or, a sermon preached at the funareall of Elizabeth Gouge, London, G. Millar, 1626, pp. 51-52. (La cursiva es propia). 